\title{
Permanent neuropsychological impairment after recurrent episodes of severe hypoglycaemia in man
}

\author{
R. Wredling ${ }^{1}$, S. Levander ${ }^{2}$, U. Adamson ${ }^{1}$ and P.E. Lins ${ }^{1}$ \\ ${ }^{1}$ Department of Medicine, Danderyd Hospital, Sweden, and ${ }^{2}$ Institute of Psychiatry, Østmarka Hospital, Trondheim, Norway
}

\begin{abstract}
Summary. Seventeen Type 1 (insulin-dependent) diabetic patients with a history of recurrent and severe hypoglycaemia and Type 1 diabetic patients with no severe hypoglycaemia were compared as regarded performances in tests of neuropsychological functioning. To test the hypothesis that recurrent severe hypoglycaemia gives rise to permanent cognitive impairment, the study group was selected among those patients who had met with repeated attacks over the last three years or more as identified by a questionnaire among almost 600 insulin-treated diabetic patients. The comparison group without known severe reactions were comparable to the study group with respect to type of diabetes, sex, age, age at onset, duration of diabetes, socio-economic parameters, and prevalence of neuropathy and retinopathy. The results indicate that Type 1 diabetic patients with recurrent severe
\end{abstract}

hypoglycaemia scored lower than those without severe hypoglycaemia in tests of motor ability, short-term and associative memory and visuospatial tasks assessing ability in general problem-solving. Type 1 diabetic patients with severe hypoglycaemia also displayed a higher frequency of perspective reversals suggesting frontal-lobe involvement. These data can be interpreted in two ways. One interpretation implies that the cognitive impairment of Type 1 diabetic patients with severe hypoglycaemia reflects a selection factor, the other that recurrent episodes of severe hypoglycaemia result in permanent cognitive impairment.

Key words: Severe hypoglycaemia, cognitive function and hypoglycaemia, Type 1 (insulin-dependent) diabetes mellitus.
Patients with Type 1 (insulin-dependent) diabetes mellitus always run the risk of suffering hypoglycaemic episodes, a fact which has become more and more evident over the last decade, as systematic monitoring of blood glucose has revealed unexpectedly high frequencies of hypoglycaemia [1-3]. Hypoglycaemia influences brain function due to the inability of the brain to utilize oxygen at low blood glucose levels, in spite of normal arterial oxygen tension [4], which may result in brain damage. In clinical medicine such damage may be evident in the form of EEG abnormalities [5-8], mild dementia [9] and permanent neurologic sequelae $[5,10]$. Children seem to be particularly vulnerable in this respect and youngsters who have had episodes of severe hypoglycaemia before the age of five show neuropsychological or EEG abnormalities with high frequency [11-13]. The majority of reports relating brain dysfunction to hypoglycaemia concern Type 1 diabetic children. So far, only a few studies have examined the relationship between hypoglycaemic episodes and brain function in adult Type 1 diabetic patients [9, 14]. These studies show that patients with a case history of severe hypoglycaemia perform more poorly than others in visuo-motor-coordination tests and that they display a lower IQ. Furthermore, some patients show mild dementia.

The present study was focussed on a subgroup of adult Type 1 diabetic patients with recurrent episodes of severe hypoglycaemia selected from a large group of insulintreated diabetic patients. Our aim was to evaluate the applicability of a battery of computerized neuropsychological tests in the present context, and to analyse whether recurrent severe hypoglycaemia could give rise to permanent cognitive impairment.

\section{Subjects and methods}

During 1985, insulin-treated Type 1 and Type 2 diabetic patients attending our out-patient clinic were asked to complete a questionnaire covering the frequency and symptoms of hypoglycaemia during the preceding 12 months. In particular, they were questioned about episodes of hypoglycaemia in which external help was re- 
Table 1. Group characteristics. Type 1 (insulin-dependent) diabetic patients with recurrent severe hypoglycaemia (SH) and without severe hypoglycaemia (no-SH)

\begin{tabular}{|c|c|c|}
\hline & $\begin{array}{l}\mathrm{SH} \\
(n=17)\end{array}$ & $\begin{array}{l}\text { no-SH } \\
(n=17)\end{array}$ \\
\hline Age of subjects (years) & $49 \pm 18$ & $48 \pm 17$ \\
\hline Onset age (years) & $20 \pm 17$ & $19 \pm 15$ \\
\hline Duration of diabetes (years) & $28 \pm 18$ & $29 \pm 13$ \\
\hline Three or more injections/day (\%) & 88 & 82 \\
\hline Insulin dose $\left(\mathrm{U} \cdot \mathrm{kg}^{-1} \cdot\right.$ day $\left.^{-1}\right)$ & $0.51 \pm 0.08$ & $0.56 \pm 0.11$ \\
\hline $\mathrm{HbA}_{\mathrm{Ic}}(\%)$ & $7.9 \pm 1.6$ & $8.8 \pm 1.6$ \\
\hline Neuropathy $(0 / 1 / 2 / 3)^{\mathrm{a}}$ & $7 / 2 / 3 / 5$ & $6 / 1 / 6 / 4$ \\
\hline Retinopathy $(0 / 1 / 2)^{b}$ & $2 / 11 / 4$ & $2 / 9 / 6$ \\
\hline
\end{tabular}

Results expressed as $n$, mean \pm SD. ${ }^{a}$ Neurological examination by physician, $0=$ no symptoms or signs, $1=$ symptomatic sensory neuropathy, $2=$ loss of deep tendon reflex, $3=1+2$. ' ${ }^{\text {F }}$ Fundoscopic examination by ophthalmologist, $0=$ none, $1=$ non-proliferative, $2=$ proliferative

quired for relief. Five hundred and thirty-three patients (90\%) out of a possible 593 answered the questionnaire. Ninety-eight of those patients $(18 \%)$ reported at least one severe hypoglycemic episode, defined as an event with unconsciousness and need for external help. A detailed analysis revealed that twenty of those 98 patients had met with repeated attacks over the last three years or more without any obvious reason. Three patients were not further investigated; one 78-year-old female patient died during the investigation due to endstage, diabetic nephropathy and two were excluded because of high age and poor general condition. The remaining 17 patients ( 8 males, 9 females) having met with recurrent severe hypoglycaemia (SH) formed the study group. All were C-peptide negative and considered as Type 1 diabetic patients. For comparison a group of 17 patients was selected by matching, in order of priority, type of diabetes, sex, age and duration of diabetes from among the remaining 435 patients who, in the above questionnaire, did not report any episodes of severe hypoglycaemia (no-SH). The two groups were comparable with respect to sex, age, age at onset, duration of diabetes, injection frequency, insulin dose and $\mathrm{HbA}_{\mathrm{lc}}$ (normal values below 5.6\%). The prevalence of neuropathy and retinopathy was also similar in the two groups (Table 1). One patient in the no-SH group had poor visual acuity due to proliferative retinopathy (visus 0.2 on the best eye) but had no difficulty participating in the test. Only a limited number of the patients were using tranquilizers, thus, among the patients with $\mathrm{SH}$, one was treated with nortriptylinchloride due to depression, another patient, suffering from insomnia, was regularly taking alimemazine and a third patient was taking hydroxizinchloride because of chronic allergic urticaria. In the no-SH group two patients were regularly taking nitrazepam due to insomnia.

When asked to participate in the study, all patients accepted. The study was approved by the local Ethics Committee and all consenting persons signed an informed consent form. Patients were instructed beforehand to keep their regular lifestyle and to monitor blood glucose during the $24 \mathrm{~h}$ prior to the test. The test was postponed if hypoglycaemia, i.e. blood glucose below $3 \mathrm{mmol} / 1$, occurred within that period. If the blood glucose level was between 3-6 mmol/l the patients were instructed to eat extra food.

In connection with the test session a personal interview about socio-economic factors was performed by using a standardized questionnaire. The employment status was studied by means of a modified version of the American Quality of Employment Survey [15]. The employment category included those who were currently employed full time or part time; the not professional practising included retired persons, housewives and students. The patients alcohol consumption was divided into five levels according to a classification based on a tentative risk evaluation scale [16].

The neuropsychological examinations were performed at about 10.00 hours under out-patient conditions. Blood glucose was measured immediately before and after the neuropsychological test session.
Computerized neuropsychological tasks

Cognitive functions were assessed by the Automated Psychological Test System [17]. The time required for a test session of eight separate tests was approximately $60 \mathrm{~min}$, including instructions. Each subject participated in two identical test sessions. Only data from the second session are presented. The following tests were administered.

The finger tapping test, assessing the motor ability, comprising five subtests: (1) right index-finger tapping, (2) left index-finger tapping, (3) right index/middle-finger alternation, (4) left index/ middle-finger alternation and (5) right/left index-finger alternation. The results are given as the number of tappings/alternations per second.

The reaction time ( $R T$ ) test, comprising four subtasks: (A) simple auditory RT: 10 auditory stimuli of $1000 \mathrm{~Hz}$, at approximately 65 decibel, (B) simple visual RT: 10 light stimuli, size $22 \times 22 \mathrm{~mm}$, in the center of the monitor screen, superimposed on a constantly displayed, central fixation cross, (C) two-choice (left-right) visual RT: 10 stimuli to the right of the center of the screen and 10 to the left in random balanced order, distanced $80 \mathrm{~mm}$ from the central fixation cross, and (D) two-choice visual stimuli with auditory signals for response inhibition: 20 visual stimuli, presented either to the right or to the left of the center of the screen, in random balanced order. Eleven of these visual stimuli were paired with a concomitant auditory signal. Such "double" stimuli were to be neglected. The following parameters were calculated (a) mean reaction time (ms) for each subtask, scparately for left-sided and right-sided stimuli in subtasks $\mathrm{C}$ and D, (b) number of correct responses to left-vs right-sided stimuli in subtask C, and (c) number of failed inhibitions of responses in subtask D.

The trail-making test, assessing visuo-motor-coordination, visual search and eye-hand coordination: The subjects were to hit digits and letters in serial order by means of a joy-stick monitored cursor. The test included three parts: (1) a training session, during which the test and its solution were demonstrated twice on letters J to $O$; (2) a session with two Form A subtests (digits only, from 1 to 9) and one Form B subtest (digits and letters in combination from $1 \mathrm{~A}$ to $9 \mathrm{~J}$ ) for the preferred hand; and (3) an identical session for the nonpreferred hand. The scores used were means of individual hit times for the two Form A subtests (18 hits) and means of the Form B subtest ( $18 \mathrm{hits)}$ for the preferred and the non-preferred hand. In addition, instances of failure to follow the sequence was assessed by a "navigation" parameter.

The Necker cube test, assessing the frequency of passively perceived perspective reversals, assumed to reflect frontal-lobe functions: Before the test started, the figure-reversal phenomenon was explained to the subjects and demonstrated by an outline of a Necker cube drawn on a card. The subjects were instructed to look steadily at a central fixation point in the middle of the cube and to press a button each time the cube changed in perspective. The Necker cube $(15 \times 15 \mathrm{~cm})$ was presented for $90 \mathrm{~s}$. The scores were the numbers of perceived reversals, the median time interval between perceived reversals, and the time from the presentation of the cube until the first perceived reversal.

The digit span test, reflecting short-term memory and concentration: The subjects were to memorize a series of digits, showing up like blips on the screen and then to reproduce them on the keyboard. In the first part this was to be done in shown order, in the second part in reverse order. The level of difficulty, i.e. the number of digits presented, was process-controlled; for each correctly reproduced item, the next contained one more digit and for each incorrectly reproduced item, the next contained one less digit. The forward task, starting with three digits, included 13 series. The backward task, starting with two digits, included 10 series. The scores were (a) the 
maximum number of digits forward, (b) the median of the last three series of digits forward, and corresponding data for digits backward.

The digit-symbol test, reflecting motor speed, visual search and associative learning: Sets of letter/digit combinations were shown on a translation board on the upper part of the screen. Then single letters were shown and the subjects were to mark corresponding digits on the keyboard. The number of sets increased stepwise from two over five to ten. Total monitoring time was $5 \mathrm{~min}$. The scores were (a) the mean response time in phases 1,2 and 3 , and (b) the number of errors in phases 1,2 and 3 .

The maze test, a rather complex, visuo-spatial task reflecting factors of general intelligence, visuo-spatial skill, motor speed, and strategy and laterality factors $[18,19]$ : Maze patterns were presented on the monitor. The task of the subjects were to complete a pathway through the maze as fast as possible, passing as many targets as possible. The path through the maze was selected by operating a set of four buttons on the keyboard. The number of targets required for a correct solution might be presented with the maze pattern (with target information) or this information could be omitted (no target information). After an introductory maze session, the subjects were given a total of 16 mazes to solve, eight with target information and eight with no target information. The following parameters, based on skill and strategy aspects [20], were calculated for each maze: processing rate (nodes per s), inspection rate (time in s from presentation of maze until first key press, divided by number of nodes of maze), check time (time after completing the pathway until pressing the confirm button), left/right preference (arbitrary units for preference for left-vs right-sided solutions), motor time (typical time for the most rapid key-pressing in $\mathrm{ms}$ ), number of correctly solved mazes, largest maze pattern solved correctly, and number of rubouts. Medians were calculated over the eight mazes within the two conditions (target information and no target information). Inspection time, check time and number of rub-outs were interpreted as strategy indices. Left/right preference was used as a laterality parameter. Processing rate, motor time and number of correctly solved mazes were skill parameters. Finally, the contrast between processing speed and number of correctly solved mazes is a strategy parameter.

The digit-symbolmemory test assessing associative memory: This test is identical to the digit-symbol test, but without the translation list and with duration time 3 min only. Subjects were instructed to respond within $6 \mathrm{~s}$ to letter stimuli according to what they remembered of the translation list and encouraged to guess when uncertain. The scores were (a) mean response time and (b) number of correct vs incorrect responses.

\section{Statistical analysis}

The results were analysed mainly by Student's $t$-test and the Wilcoxon paired-rank sum test when applicable. To assess differences in alcohol intake the Mann-Whitney sum rank test was used. Probabilities below $p<0.05$ were regarded as statistically significant. Unless otherwise stated, the data are presented as mean $\pm \mathrm{SD}$.

\section{Results}

The mean blood glucose concentrations before and after the neuropsychological testing were $10.7 \pm 4.9 \mathrm{mmol} / \mathrm{l}$ (range 3.5-19.6) and $13.2 \pm 3.5 \mathrm{mmol} / 1$ (range $7.7-19.3$ ) in Type 1 patients with $\mathrm{SH}$, and $10.1 \pm 5.4 \mathrm{mmol} / \mathrm{l}$ (range 4.4-21.3) and $9.9 \pm 5.7 \mathrm{mmol} / \mathrm{l}$ (range 4.1-23.3) in Type 1 patients with no-SH, respectively. Two patients with $\mathrm{SH}$
Table 2. Education, employment and alcohol consumption. Type 1 (insulin-dependent) diabetic patients with recurrent severe hypoglycaemia $(\mathrm{SH})$ and without severe hypoglycaemia (no-SH)

\begin{tabular}{lll}
\hline & $\begin{array}{l}\text { SH } \\
(n=17)\end{array}$ & $\begin{array}{l}\text { no-SH } \\
(n=17)\end{array}$ \\
\hline Education & 3 & 3 \\
$\quad$ Primary school (7 years) & 2 & 1 \\
Primary school (9 years) & 8 & 9 \\
$\quad$ College (12 years) & 4 & 4 \\
$\quad$ University ( $>12$ years) & & \\
Employment status & 3 & 5 \\
Full-time work $>35$ hours/week & 6 & 4 \\
Part-time work $\leq 35$ hours/week & 8 & 8 \\
$\quad$ Not professional practising & & \\
Level of exertion & 5 & 2 \\
Blue collar workers & 12 & 15 \\
White collar workers & & \\
Alcohol consumption & $5 / 11 / 1 /-1-$ & $1 / 12 / 1 / 2 / 1$ \\
0/1/2/3/4a
\end{tabular}

Results expressed as $n$. $0=$ no alcohol consumption during the preceeding $12 \mathrm{months,} 1=\leq 50 \mathrm{~g}$ alcohol $/$ week, $2=\leq 110 \mathrm{~g}$ alco$\mathrm{hol} / \mathrm{week}, 3=\leq 250 \mathrm{~g}$ alcohol $/$ week, $4=\leq 400 \mathrm{~g}$ alcohol $/$ week

and one with no-SH had blood glucose values between 3.5 and $4.4 \mathrm{mmol} / \mathrm{l}$ before the test and were, therefore, given extra food to prevent hypoglycaemia during the test procedure.

Socio-economic parameters are shown in Table 2. The two groups were comparable with respect to education, employment status and level of exertion. No significant difference in alcohol consumption between the groups was found $(23.5 \pm 22.4$ vs $62.3 \pm 89.7 \mathrm{~g}$ alcohol/week). However, the tendency in the SH group was toward a greater number of teetotallers. Also in this group no patient was consuming more than the average intake of alcohol among Swedish adult inhabitants, $96 \mathrm{~g}$ alcohol/week [21].

The neuropsychological test scores are given in Table 3. Data from the finger tapping test, assessing the motor ability, showed significantly slower tapping in alternation right $(t=2.17, p<0.05)$ and left $(t=2.30, p<0.05)$ for Type 1 diabetic patients with SH.

Data from the Necker cube test, assessing perspective reversals, showed a significantly higher number of perceived reversals among Type 1 diabetic patients with $\mathrm{SH}$ $(t=2.06, p<0.05)$.

Short-term memory and concentration, as assessed by the digit span test, showed that Type 1 diabetic patients with $\mathrm{SH}$ were not able to repeat as many number of digits forward as Type 1 diabetic patients with no-SH ( $t=2.06$, $p<0.05)$. Associative memory, as assessed by the digitsymbol test, showed that Type 1 diabetic SH patients responded slower in the task of translating letters into digits, when the translation list comprised five letters ( 2493 vs $2186 \mathrm{~ms}, t=2.12, p<0.05)$. There was also a trend towards significance in response time when ten letter/digit pairs were presented, Type 1 diabetic SH patients responding slower (2832 vs $2545 \mathrm{~ms}, t=1.90, p<0.10$ ). In the digit-symbol memory test, no significant differences between the groups were found. 
Table 3. Neuropsychological tests for Type 1 (insulin-dependent) diabetic patients with recurrent severe hypoglycaemia $(\mathrm{SH})$ and no severe hypoglycaemia (no-SH) respectively

\begin{tabular}{|c|c|c|}
\hline & $\begin{array}{l}\text { SH } \\
(n=17)\end{array}$ & $\begin{array}{l}\text { no-SH } \\
(n=17)\end{array}$ \\
\hline \multicolumn{3}{|l|}{ Finger tapping test $(\# / s)$} \\
\hline $\begin{array}{l}\text { Tapping with index finger } \\
\text { Right } \\
\text { Left }\end{array}$ & $\begin{array}{l}5.9 \pm 1.1 \\
5.3 \pm 1.0\end{array}$ & $\begin{array}{l}5.9 \pm 1.5 \\
5.7 \pm 0.9\end{array}$ \\
\hline $\begin{array}{l}\text { Alternation } \\
\text { Right } \\
\text { Left }\end{array}$ & $\begin{array}{l}2.7 \pm 1.2 \\
2.3 \pm 1.2\end{array}$ & $\begin{array}{l}3.7 \pm 1.3^{c} \\
3.3 \pm 1.3^{c}\end{array}$ \\
\hline Alternation right/left & $3.1 \pm 0.9$ & $3.5 \pm 1.0$ \\
\hline \multicolumn{3}{|l|}{ Necker cube } \\
\hline Perceptual shifts (\#) & $9.4 \pm 7.3$ & $5.9 \pm 3.9^{c}$ \\
\hline \multicolumn{3}{|l|}{ Digitspan (\#) } \\
\hline Maximum $n$ of digits forward & $7.2 \pm 1.2$ & $7.8 \pm 1.2^{c}$ \\
\hline $\begin{array}{l}\text { Median of last } 3 \text { series of digits } \\
\text { forward }\end{array}$ & $5.9 \pm 1.4$ & $6.4 \pm 1.1$ \\
\hline Maximum $n$ of digits backward & $6.3 \pm 1.4$ & $6.7 \pm 1.4$ \\
\hline $\begin{array}{l}\text { Median of last } 3 \text { series of digits } \\
\text { backward }\end{array}$ & $5.5 \pm 1.8$ & $5.8 \pm 1.7$ \\
\hline \multicolumn{3}{|l|}{ Maze test (with target information) } \\
\hline Processing rate (nodes/s) & $3.0 \pm 2.2$ & $4.4 \pm 2.4^{a}$ \\
\hline Inspection rate (nodes/s) & $37.9 \pm 16.9$ & $36.9 \pm 16.3$ \\
\hline Check time(s) & $0.9 \pm 0.3$ & $0.7 \pm 0.2^{b}$ \\
\hline L/R preference (arbitrary unit) & $0.5 \pm 0.2$ & $0.5 \pm 0.2$ \\
\hline Motor time (ms) & $471 \pm 147$ & $442 \pm 145$ \\
\hline Correctly solved mazes (\%) & $82 \pm 15$ & $70 \pm 23^{c}$ \\
\hline $\begin{array}{l}\text { Maximum size of a correctly solved } \\
\text { maze (rows) }\end{array}$ & $10.3 \pm 2.4$ & $11.4 \pm 3.7$ \\
\hline Rub-outs (\#/maze) & $0.7 \pm 0.6$ & $0.7 \pm 0.8$ \\
\hline
\end{tabular}

Significant difference from the SH group; ${ }^{\mathrm{a}} p<0.001 ;{ }^{\mathrm{b}} p<0.01$;

${ }^{c} p<0.05 ;$ Results expressed as mean \pm SD

In the reaction time assessment, there was a trend suggesting that Type 1 diabetic SH patients elicited more crossed responses (left-sided response to a right-sided stimulus and vice versa) in the two-choice reaction time task ( 2.4 vs $1.7, t=1.69, p<0.10)$. There were no other differences in the reaction time task between the groups. Neither were there any differences for the trail-making test parameters.

The most complex test used in our study, the maze test, was applied in order to assess general problem-solving ability. Data from this test showed that Type 1 diabetic patients with $\mathrm{SH}$ had a markedly slower processing rate $(t=3.29, p<0.001)$ and needed more time between the solution and the confirmation of each problem $(t=2.29$, $p<0.01$ ). Type 1 diabetic SH patients also used a more cautious strategy and therefore solved more mazes correctly $(t=2.31, p<0.05)$. The performance on the maze test condition without target information showed that Type 1 diabetic patients with SH used a more sequential, left-hemisphere-type strategy in the solving process by using fewer rub-outs $(0.3$ vs 0.7 per maze, $t=3.01$, $p<0.01)$.

\section{Discussion}

The present study supports the conclusion in the previously cited earlier (1973) study by Bale [9] that brain damage is related to the severity of past hypoglycaemic episodes. Our study is also in line with previous research by Skenazy [14] which found that performance IQ correlated negatively with the incidence of severe hypoglycaemic reactions. Bale assessed cognitive functions by the Walton-Black new world learning test $[22,23]$ and the Wechsler adult intelligence scale [24]. Skenazy, in 1984, also assessed cerebral faculties by the Wechsler adult intelligence scale [24] and in addition by the Halstead-Reitan neuropsychological test battery [25]. In our study we employed a computerized neuropsychological test battery developed in the 1980's [26].

It is well known that the cognitive capacity is reduced acutely by hypoglycaemia, alcohol and many drugs. Also the chronic use of alcohol may affect intellectual functions. In the present study we had no indication that the diabetic patients with $\mathrm{SH}$ were using alcohol or tranquilizers more extensively, and during the test situation they all had a blood glucose level above the hypoglycaemic range. The difference observed in the cognitive tests between our two groups of Type 1 diabetic patients was probably not attributed to late diabetic complications as tests like reaction time, trail-making i.e. tests that required motor strength, motor speed and visual efficiency, were close to identical in the two groups. We also estimate that the reduction in cognitive capacity was acquired during their life with the disease, as the two groups had the same educational level.

Three of the neuropsychological methods used in our study are assumed to reflect frontal-lobe functions, on different levels of complexity and integration, the finger tapping, the Necker cube and the maze tests. The frontal lobes are concerned with planning, programming and controlling actions [20]. Speed of finger alternation, a part of the finger tapping test which measures a moderately complex motor act, was significantly impaired in our Type 1 diabetic patients with SH. The Necker cube data demonstrated that these patients had a significantly higher number of perspective reversals than those without severe hypoglycaemia. According to Cohen [27], unilateral damage decreases and bilateral damage increases the rate of such reversals, and in a study by Yacorzinski and Davies it was demonstrated that patients with unilateral frontal lobectomy perceived fewer reversals than the control subjects [28]. During acute hypoglycaemia, i.e. blood glucose concentration of between 1.8 and $2.9 \mathrm{mmol} / 1$ executive cognitive functions were profoundly affected and the frontal-lobe based functions were unilaterally affected [29]. Our Necker cube and finger tapping results, thus, suggest that the frontal-lobe dysfunction related to hypoglycaemic episodes is bilateral.

Performance in the maze tasks showed that the Type 1 diabetic patients with no-SH were superior to the patients with $\mathrm{SH}$, particularly for mazes with target information. The maze test was originally designed to assess frontal lobe functions, but empirical studies, as well as a 
content analysis of the test suggest that factors of visuospatial skill, general intelligence and presence of brain lesion have high loadings [18]. Our data suggest that Type 1 diabetic patients with SH differed from the comparison group in two ways: they displayed inferior performance and they solved the test in a different way. Their solution strategy was characterized by a marked preference for accuracy rather than speed, and by doubts and hesitation when acknowledging the solution. Such a pattern of slowness and over-control has been associated with lateral rather than orbitomedial frontal lobe lesions [30]. One may speculate on how this particular way of solving a complex psychological test generalizes to problem-solving situations in everyday life. It is not too far fetched to suggest that indecision and anxiety will characterize such patients when confronted with a situation that calls for fast decisions. We have frequently observed that diabetic patients with recurrent severe hypoglycaemia, i.e. despite their knowledge and awareness of high-risk situations that might result in hypoglycaemia, do not take the necessary steps to avoid such situations.

Type 1 diabetic patients with SH showed slight impairments of short-term and associative memory in our study. These findings are in line with those of Bale, who showed that patients with SH performed poorly in the tests of auditory learning, including short-term memory [9]. In addition, we found a non-significant tendency towards deterioriated memory performances in our hypoglycaemiaprone patients concerning all the memory tasks applied. These memory disturbances may affect the activities of daily life in these patients in a similar manner to that of premature aging.

Our study shows that Type 1 diabetic patients with SH are cognitively impaired when compared with Type 1 diabetic patients with no-SH. The two groups were comparable with respect to sex, age, age at onset, duration of diabetes, educational level and alcohol consumption, a number of parameters which otherwise would have confounded the differences. Part of the impairment appears to be of the type associated with organic brain damage, and part appears to be related to preference for particular problem solving strategies, but it is impossible to say whether that behaviour is the cause for or the effect of recurrent severe hypoglycaemia. Since our study is retrospective, there are two possible interpretations of the association between hypoglycaemic episodes and cognitive impairment. Either there is a causal link, i.e. repeated severe hypoglycaemic episodes cause permanent cognitive impairment, or the association reflects some kind of selection procedure or a common underlying factor. This factor should then be associated both with an increased frequency of hypoglycaemia, and with a lower cognitive performance level.

The two groups of patients were almost identical in terms of level of education, suggesting that there was probably no great difference in premorbid intellectual level between the groups. Compared to healthy control subjects the performance of the two groups of the present study was inferior in many of the tests, and more so for the SH group. This is probably related to age. The com- parison group which is avaliable has a median age of 35 (range 20 to 50 ) in contrast to the present one, in which age ranged from 26 to 72 with a median of 49 . It is not easy to apply a meaningful comparison at a group level with such a large age span. Instead, we used an agematching paradigm, which is a more effective way to correct for the age factor.

In conclusion, since the study is retrospective, the data can be interpreted in two ways. One interpretation implies that the cognitive impairment of adult Type 1 diabetic patients with $\mathrm{SH}$ reflects a selection factor, the other that recurrent episodes of SH result in permanent cognitive impairment.

Acknowledgements. This work was supported by grants from the Stockholm County Council, the Swedish Medical Research Council (19x-6589), the Karolinska Institute, the Swedish Hoechst Diabetes Foundation, the Nordic Insulin Foundation and the Novo Industries A/S.

\section{References}

1. Pramming S, Thorsteinsson B, Bendtson I, Rönn B, Binder C (1985) Nocturnal hypoglycemia in patients receiving conventional treatment with insulin. Br Med J 291:376-379

2. Cox JD, Clarke LW, Gonder-Frederick L, Pohl S, Hoover C, Snyder A, Zimbelman L, Carte RW, Robbitt S, Pennabaker J (1985) Accuracy of perceiving blood glucose in IDDM. Diabetes Care 6: 529-536

3. Gale EAM, Tattersall RB (1979) Unrecognized nocturnal hypoglycemia in insulin-treated diabetics. Lancet I: 1049-1052

4. Hirabayashi S, Kitahara T, Hishida T (1980) Computed tomography in perinatal hypoxic and hypoglycemic encephalopathy with emphasis on follow-up studies. J Comput Assist Tomogr 4: 451-456

5. Ingram TTS, Stark GD, Blackburn I (1967) Ataxia and other neurological disorders as sequels of severe hypoglycaemia in childhood. Brain 90: 851-862

6. Haumont D, Dorchy H, Pelc S (1979) EEG abnormalities in diabetic children: influence of hypoglycemia and vascular complications. Clin Pediatr 18: 750-753

7. Gilhaus KH, Daweke H, Lülsdorf HG, Sachsse R, Sachsse B (1973) EEG-Veränderungen bei diabetischen Kindern. Dtsch Med Wochenschr 98: 1449-1454

8. Eeg-Olofsson O, Petersén I (1966) Childhood diabetic neuropathy: a clinical and neuropsychological study. Acta Paediatr Scand 55: 163-176

9. Bale RN (1973) Brain damage in diabetes mellitus. Br J Psychiat 122: $337-341$

10. Hawort JC, Coodin JF (1960) Idiopathic spontaneous hypoglycemia in children. Pediatrics 25:748-765

11. Ryan C, Vega A, Drash A (1985) Cognitive deficits in adolescents who developed diabetes early in life. Pediatrics 75: 921-927

12. Rovet FJ, Ehrlich MR, Hoppe M (1987) Intellectual deficits associated with early onset of insulin-dependent diabetes mellitus in children. Diabetes Care 4: 510-515

13. Ack M, Miller I, Weil BW (1961) Intelligence of children with diabetes mellitus. Pediatrics 28: 764-770

14. Skenazy AJ, Bigler DE (1984) Neuropsychological findings in diabetes mellitus. J Clin Psychol 1: 246-258

15. Karasek RA, Schwartz J, Theorell T (1982) Job characteristics, occupation and coronary heart disease. Dept Industrial Engin and Operation Research, Columbia Univ, New York (Stencile)

16. Rydberg U (1977) The toxicity of ethanol. A tentative risk evaluation. In: Gross MM (ed) Alcohol intoxication and withdrawal, Vol 3 B. Plenum, New York, pp 403-414 
17. Levander SE, Elithorn A (1987) An automated psychological test system. APT Manual. Department of Psychiatry and Behavioral Medicine, University of Trondheim

18. Elithorn A, Powell J, Telford A (1976) Mental assessment on line. Proc Electronic Display 3: 18-25

19. Elithorn A (1955) A preliminary report on a perceptual maze test sensitive to brain damage. J Neurol Neurosurg Psychiatry 18 : 287-292

20. Luria AR (1980) Higher cortical function in man. 2nd edn. Basic Books, New York

21. National Board of Health and Welfare (1988) Alkoholstatistik 1988. National Board of Health and Welfare, Stockholm

22. Walton D, Black DA (1957) The validity of a psychological test of brain damage. Br J Med Psychol 30: 270-279

23. Walton D, Black DA (1959) The predictive validity of a psychological test of brain damage. J Ment Sci 105: 807-810

24. Wechsler D (1955) Manual for the Wechsler adult intelligence scale. The Psychological Corporation, New York

25. Reitan R (1955) An investigation of the validity of Halstead's measures of biological intelligence. Archives Neurol Psychiat 73: 28-35
26. Levander S (1988) Evaluation of cognitive impairment using a computerized neuropsychological test battery. J Clin Exp Neuropsychol 10: 3 (Abstract)

27. Cohen L (1959) Perception of reversible figures after brain injury. Archives Neurol Psychiat 81: 765-775

28. Yacorzynski GK, Davis L (1945) An experimental study of the function of the frontal lobes in man. Psychosom Med 7: 97-107

29. Pramming S, Thorsteinsson B, Theilgaard A, Pinner EM, Binder C (1986) Cognitive function during hypoglycaemia in type I diabetes mellitus. Br Med J 292: 647-650

30. Stuss DT, Benson DF (1986) The frontal lobes. Raven Press, New York

Received: 2 June 1989

and in revised form: 29 September 1989

Ms. R. Wredling

Department of Medicine

Danderyd Hospital

S-18288 Danderyd

Sweden 